Supplement of Hydrol. Earth Syst. Sci., 25, 89-104, 2021

https://doi.org/10.5194/hess-25-89-2021-supplement

(c) Author(s) 2021. This work is distributed under

the Creative Commons Attribution 4.0 License.

(c) (1)

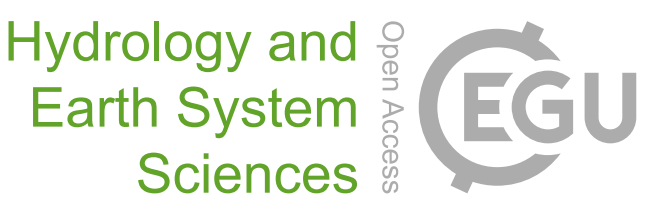

Supplement of

\title{
Using multiple methods to investigate the effects of land-use changes on groundwater recharge in a semi-arid area
}

Shovon Barua et al.

Correspondence to: Shovon Barua (shovon.barua@monash.edu)

The copyright of individual parts of the supplement might differ from the CC BY 4.0 License. 
Supplement Table S1: Geochemistry and hydraulic conductivities of groundwater at Gatum.

\begin{tabular}{|c|c|c|c|c|c|c|c|c|c|c|c|c|c|c|c|c|c|c|}
\hline $\begin{array}{c}\text { Sampling } \\
\text { date }\end{array}$ & Sample & $\begin{array}{c}\text { Sample } \\
\operatorname{depth}(\mathbf{m})\end{array}$ & $\begin{array}{l}\text { Landscape } \\
\text { position }\end{array}$ & $\begin{array}{c}\mathbf{K}_{\mathrm{s}} \\
\mathbf{m}_{\text {day }}{ }^{-1}\end{array}$ & $\begin{array}{c}\mathrm{EC} \\
\mu \mathrm{S} \mathbf{c m}^{-1}\end{array}$ & TDS & $\mathrm{Na}$ & Mg & $\mathrm{Ca}$ & $\mathbf{S i}$ & $\begin{array}{c}\mathbf{K} \\
\mathbf{m g ~ L ^ { - 1 }}\end{array}$ & Cl & $\mathrm{SO}_{4}$ & $\mathrm{HCO}_{3}$ & $\mathrm{NO}_{3}$ & $\mathrm{Br}$ & $\begin{array}{l}{ }^{14} \mathrm{C} \\
\mathrm{pMC}\end{array}$ & $\begin{array}{l}{ }^{3} \mathrm{H} \\
\mathrm{TU}\end{array}$ \\
\hline \multicolumn{19}{|l|}{ Pasture Catchment } \\
\hline $23 / 05 / 2018$ & 63 & 15.5 & Upper & 0.31 & 442 & 282 & 62.8 & 3.19 & 0.56 & 13.6 & 1.21 & 45.2 & 27.9 & 68.3 & 59.3 & 0.16 & $104 \pm 0.35$ & $1.48 \pm 0.03$ \\
\hline $23 / 05 / 2018$ & 3013 & 19.2 & Upper & & 5770 & 3930 & 1200 & 162 & 84.6 & 26.4 & 8.89 & 2210 & 208 & 13.4 & 14.3 & 6.29 & $92.0 \pm 0.32$ & $0.50 \pm 0.02$ \\
\hline $1 / 11 / 2018$ & 3013 & 19.2 & Upper & & 4850 & 3390 & 1010 & 160 & 80.6 & 24.7 & 8.67 & 1830 & 176 & 56.1 & 38.6 & 5.43 & & \\
\hline $24 / 05 / 2018$ & 3001 & 10.7 & Mid & 0.06 & 9390 & 6380 & 1650 & 346 & 164 & 37.7 & 7.26 & 3810 & 314 & 42.7 & 1.65 & 10.3 & $77.6 \pm 0.27$ & $0.25 \pm 0.02$ \\
\hline $24 / 05 / 2018$ & 3002 & 8.0 & Mid & 0.08 & 9590 & 6370 & 1670 & 258 & 198 & 32.2 & 10.8 & 3720 & 298 & 173 & 2.19 & 10.4 & $84.5 \pm 0.29$ & $0.02 \pm 0.01$ \\
\hline $24 / 05 / 2018$ & 3004 & 10.6 & Mid & 0.07 & 6410 & 4090 & 1190 & 143 & 142 & 28.9 & 4.05 & 2300 & 229 & 37.8 & 7.40 & 7.39 & $72.3 \pm 0.27$ & $0.13 \pm 0.02$ \\
\hline $23 / 05 / 2018$ & 3019 & 13.3 & Mid & 0.11 & 3680 & 2100 & 686 & 70.9 & 14.3 & 34.8 & 7.17 & 1130 & 88.0 & 52.5 & 17.2 & 3.42 & $89.5 \pm 0.31$ & $0.72 \pm 0.02$ \\
\hline $23 / 05 / 2018$ & 3007 & 17.0 & Drainage & 0.08 & 7700 & 5210 & 1450 & 238 & 40.8 & 33.1 & 15.6 & 3110 & 260 & 47.6 & 2.16 & 9.11 & $92.9 \pm 0.32$ & $0.08 \pm 0.02$ \\
\hline $7 / 09 / 2018$ & 3008 & 1.3 & Drainage & & 7640 & 7850 & 2410 & 396 & 125 & 20.0 & 0.16 & 4570 & 305 & 1.22 & 6.56 & 13.8 & $98.3 \pm 0.30$ & $1.10 \pm 0.03$ \\
\hline $3 / 07 / 2018$ & $P D 1$ & 1 & Drainage & & 3940 & 4130 & 1250 & 181 & 36.2 & 106 & 11.4 & 2340 & 189 & 11.0 & 1.15 & 7.09 & $91.1 \pm 0.29$ & $0.16 \pm 0.02$ \\
\hline $7 / 09 / 2018$ & $P D 1$ & 1 & Drainage & & 6710 & 3890 & 1200 & 166 & 31.5 & 37.6 & 9.22 & 2230 & 180 & 24.4 & 1.26 & 6.64 & & \\
\hline $1 / 11 / 2018$ & PDI & 1 & Drainage & & 5150 & 4010 & 1230 & 181 & 34.4 & 40.1 & 10.5 & 2270 & 185 & 43.9 & 5.02 & 6.79 & & \\
\hline $3 / 07 / 2018$ & $P D 2$ & 1 & Drainage & & 8740 & 6100 & 1850 & 267 & 88.8 & 29.3 & 12.3 & 3430 & 299 & 110 & 2.86 & 10.2 & $88.2 \pm 0.33$ & $0.26 \pm 0.02$ \\
\hline $7 / 09 / 2018$ & $P D 2$ & 1 & Drainage & & 6970 & 3960 & 1210 & 169 & 48.7 & 29.8 & 8.49 & 2270 & 189 & 30.5 & 1.36 & 6.74 & & \\
\hline $1 / 11 / 2018$ & $P D 2$ & 1 & Drainage & & 5800 & 4640 & 1390 & 198 & 44.3 & 37.8 & 11.4 & 2680 & 223 & 37.8 & 5.84 & 8.19 & & \\
\hline $24 / 05 / 2018$ & 64 & 29.7 & Lower & 0.31 & 9410 & 6390 & 1490 & 348 & 244 & 36.0 & 11.1 & 3790 & 338 & 121 & 1.55 & 12.3 & $70.7 \pm 0.28$ & $b d l(0.02)$ \\
\hline $3 / 07 / 2018$ & $P B 1$ & 1 & Lower & & 6520 & 7460 & 1830 & 442 & 365 & 17.4 & 1.78 & 4390 & 364 & 35.4 & 3.25 & 14.0 & $86.0 \pm 0.28$ & $0.79 \pm 0.02$ \\
\hline 6/09/2018 & $P B 1$ & 1 & Lower & & 10100 & 7240 & 1830 & 392 & 312 & 25.8 & 0.13 & 4240 & 405 & 19.5 & 2.33 & 13.2 & & \\
\hline $1 / 11 / 2018$ & $P B 1$ & 1 & Lower & & 8750 & 7700 & 1930 & 441 & 339 & 29.3 & 0.85 & 4400 & 438 & 101 & 8.10 & 14.2 & & \\
\hline $6 / 09 / 2018$ & $P B 2$ & 1 & Lower & & 10000 & 7260 & 1980 & 320 & 270 & 12.1 & 2.88 & 4140 & 439 & 79.3 & 2.12 & 12.3 & $85.5 \pm 0.28$ & $0.69 \pm 0.02$ \\
\hline $1 / 11 / 2018$ & $P B 2$ & 1 & Lower & & 9660 & 8180 & 2260 & 401 & 342 & 18.1 & 2.31 & 4570 & 451 & 115 & 5.17 & 13.8 & & \\
\hline \multicolumn{19}{|l|}{ Forest Catchment } \\
\hline $23 / 05 / 2018$ & 3662 & 16.9 & Upper & 0.002 & 7210 & 5270 & 1270 & 398 & 192 & 31.3 & 8.57 & 3040 & 299 & 1.22 & 24.4 & 10.1 & $94.7 \pm 0.32$ & $1.15 \pm 0.03$ \\
\hline $22 / 05 / 2018$ & 3663 & 24.8 & Upper & 0.13 & 7150 & 5200 & 1490 & 329 & 62.1 & 37.7 & 21.6 & 2970 & 273 & 1.22 & 7.29 & 9.40 & $89.9 \pm 0.31$ & $0.09 \pm 0.01$ \\
\hline $22 / 05 / 2018$ & 3665 & 13.0 & Upper & 0.18 & 4960 & 3920 & 1170 & 192 & 48.8 & 35.9 & 11.8 & 2250 & 195 & 1.22 & 6.97 & 6.30 & $93.7 \pm 0.32$ & $0.05 \pm 0.02$ \\
\hline $31 / 10 / 2018$ & 3665 & 13.0 & Upper & & 5120 & 3830 & 1140 & 189 & 49.1 & 29.7 & 11.5 & 2200 & 192 & 1.22 & 13.9 & 6.14 & & \\
\hline $22 / 05 / 2018$ & 3668 & 28.4 & Mid & 0.18 & 3060 & 1840 & 438 & 87.2 & 119 & 28.5 & 12.3 & 794 & 72.2 & 288 & 2.26 & 2.52 & $29.5 \pm 0.35$ & $0.02 \pm 0.02$ \\
\hline $22 / 05 / 2018$ & 3658 & 15.8 & Drainage & 0.007 & 1430 & 1060 & 196 & 43.9 & 58.9 & 2.88 & 7.68 & 248 & 64.0 & 432 & 1.86 & 0.65 & $82.9 \pm 0.30$ & $1.43 \pm 0.04$ \\
\hline $31 / 10 / 2018$ & 3658 & 15.8 & Drainage & & 1750 & 1190 & 260 & 65.0 & 85.3 & 11.7 & 9.04 & 330 & 79.7 & 339 & 3.45 & 0.84 & & \\
\hline $22 / 05 / 2018$ & 3666 & 28.0 & Drainage & 0.009 & 7510 & 5810 & 1180 & 335 & 251 & 27.8 & 20.9 & 3510 & 461 & 15.9 & 1.82 & 9.85 & $38.1 \pm 0.32$ & $b d l(0.02)$ \\
\hline $22 / 05 / 2018$ & 3667 & 18.0 & Drainage & 0.18 & 7520 & 5460 & 1230 & 319 & 217 & 31.1 & 20.6 & 3220 & 415 & 1.22 & 1.21 & 8.87 & $53.3 \pm 0.29$ & $b d l(0.02)$ \\
\hline 6/09/2018 & 3669 & 9.0 & Drainage & & 5220 & 3440 & 1010 & 146 & 73.0 & 30.2 & 8.15 & 1930 & 201 & 30.5 & 4.55 & 5.71 & $89.4 \pm 0.29$ & $0.03 \pm 0.02$ \\
\hline $31 / 10 / 2018$ & 3669 & 9.0 & Drainage & & 4710 & 3460 & 1030 & 148 & 73.0 & 32.2 & 8.85 & 1910 & 199 & 45.1 & 8.29 & 5.71 & & \\
\hline $7 / 09 / 2018$ & $F D 1$ & 1 & Drainage & & 1210 & 746 & 210 & 17.3 & 14.6 & 18.1 & 0.82 & 311 & 109 & 61.0 & 2.06 & 1.48 & & \\
\hline $6 / 09 / 2018$ & $F D 2$ & 1 & Drainage & & 257 & 169 & 37.8 & 4.24 & 2.89 & 18.0 & 1.25 & 47.4 & 27.3 & 25.6 & 4.30 & 0.59 & $102 \pm 0.32$ & $4.10 \pm 0.06$ \\
\hline 7/09/2018 & $F D 3$ & 1 & Drainage & & 3600 & 2060 & 680 & 43.6 & 39.7 & 17.6 & 1.64 & 1030 & 103 & 139 & 2.55 & 3.44 & & \\
\hline $7 / 09 / 2018$ & $F D 4$ & 1 & Drainage & & 3240 & 2220 & 595 & 57.1 & 59.9 & 20.7 & 0.61 & 949 & 162 & 366 & 1.25 & 5.47 & $91.1 \pm 0.29$ & $2.63 \pm 0.05$ \\
\hline $31 / 10 / 2018$ & $F D 4$ & 1 & Drainage & & 3790 & 2490 & 744 & 82.6 & 76.5 & 14.8 & 0.83 & 1220 & 178 & 161 & 4.56 & 5.80 & & \\
\hline $22 / 05 / 2018$ & 3656 & 28.8 & Lower & 0.002 & 9830 & 7070 & 1680 & 394 & 288 & 19.5 & 19.8 & 4180 & 344 & 128 & 2.68 & 11.1 & $89.9 \pm 0.30$ & $1.01 \pm 0.03$ \\
\hline 6/09/2018 & 3657 & 2.5 & Lower & & 8210 & 5780 & 1490 & 298 & 225 & 18.4 & 0.98 & 3290 & 384 & 54.9 & 6.21 & 8.87 & $101 \pm 0.31$ & $2.14 \pm 0.04$ \\
\hline $31 / 10 / 2018$ & 3657 & 2.5 & Lower & & 8030 & 6850 & 1850 & 429 & 157 & 22.4 & 1.55 & 3930 & 412 & 28.1 & 7.50 & 10.6 & & \\
\hline 7/09/2018 & $F B 1$ & 1 & Lower & & 253 & 339 & 35.9 & 5.56 & 7.09 & 3.21 & 4.84 & 56.8 & 5.69 & 220 & 0.19 & 0.16 & & \\
\hline 7/09/2018 & $F B 2$ & 1 & Lower & & 1550 & 868 & 275 & 11.2 & 7.49 & 14.2 & 2.08 & 411 & 100 & 42.7 & 1.33 & 2.04 & & \\
\hline $31 / 10 / 2018$ & $F B 2$ & 1 & Lower & & 2470 & 1680 & 521 & 36.4 & 24.8 & 14.7 & 4.47 & 863 & 190 & 18.3 & 1.57 & 3.73 & & \\
\hline $3 / 07 / 2018$ & $F B 3$ & 1 & Lower & & 11800 & 8290 & 2230 & 442 & 250 & 13.9 & 6.08 & 4750 & 483 & 97.6 & 3.78 & 13.7 & & $2.01 \pm 0.04$ \\
\hline 6/09/2018 & $F B 3$ & 1 & Lower & & 14000 & 10000 & 2580 & 550 & 339 & 12.6 & 3.95 & 5820 & 571 & 96.4 & 18.6 & 16.5 & & \\
\hline $31 / 10 / 2018$ & $F B 3$ & 1 & Lower & & 13700 & 13600 & 3300 & 759 & 462 & 16.4 & 4.64 & 8140 & 781 & 80.5 & 16.7 & 23.4 & & \\
\hline $3 / 07 / 2018$ & $F B 4$ & 1 & Lower & & 11700 & 7850 & 2070 & 492 & 266 & 9.29 & 10.6 & 4580 & 365 & 36.6 & 10.6 & 13.6 & & \\
\hline 7/09/2018 & $F B 4$ & 1 & Lower & & 6830 & 4180 & 1110 & 239 & 133 & 6.66 & 4.72 & 2420 & 179 & 75.6 & 1.32 & 6.85 & & \\
\hline $1 / 5 / 2018$ to $31 / 05 / 2019$ & Rainfall & & & & & & & & & & & & & & & & & $2.79 \pm 0.05$ \\
\hline
\end{tabular}

Upper, Mid, and Lower slopes as discussed in text. Sample depth is the middle of the screened interval. bdl = below detection limit. The yearly ${ }^{3} \mathrm{H}$ value of rainfall is the aggregate value. 